\title{
Is there a distinct phenotype of diabetic patients who benefit from tofogliflozin?
}

Dimitrios Ioannis Patoulias ${ }^{1,2}$

${ }^{1}$ Department of Internal Medicine, General Hospital of Veria, Veria, Greece ${ }^{2} 2^{\text {nd }}$ Propedeutic Department of Internal Medicine, Aristotle University of Thessaloniki, General Hospital Hippokration, Thessaloniki, Greece

Submitted: 20 February 2018

Accepted: 13 March 2018

Arch Med Sci Atheroscler Dis 2018; 3: e58-e59

DOI: https://doi.org/10.5114/amsad.2018.74776

Copyright @ 2018 Termedia @ Banach

There is an increasing amount of data on the use of sodium-glucose co-transporter type 2 (SGLT-2) inhibitors in patients with type 2 diabetes, especially those at high cardiovascular risk, as they significantly improve the main cardiometabolic parameters [1, 2].

One of those highly selective SGLT-2 inhibitors is tofogliflozin. A previous 8-week open label study involving 17 Japanese type 2 diabetic patients with baseline body mass index (BMI) $28.9 \pm 4.6 \mathrm{~kg} / \mathrm{m}^{2}$ and hemoglobin glycated $\left(\mathrm{HbA}_{1 \mathrm{c}}\right.$ ) levels $7.8 \pm 1.5 \%$ showed improved glycemic control and significantly decreased body weight, BMI and free fat mass, without the total fat mass being significantly affected [3]. In another 24-week study involving patients with similar baseline characteristics, it was found that tofogliflozin produced statistically significant results in terms of decreases in $\mathrm{HbA}_{1 \mathrm{c}}$ levels, body weight and total daily insulin dose, which were observed early during the study and were maintained until the end of the trial [4].

A recently published multicenter, 52-week, phase 4 study evaluated the long-term effect of tofogliflozin as add-on therapy in Japanese patients with type 2 diabetes with sub-optimal glycemic control, receiving either insulin monotherapy or insulin plus dipeptidyl peptidase-4 (DPP-4) inhibitor [5]. Mean baseline BMI was $25.79 \pm 3.46 \mathrm{~kg} / \mathrm{m}^{2}$ for patients who received tofogliflozin throughout the study and $26.89 \pm 3.88 \mathrm{~kg} / \mathrm{m}^{2}$ for patients who received placebo (16 weeks) and then tofogliflozin (36 weeks). Mean baseline $\mathrm{HbA}_{1 \mathrm{c}}$ levels were $8.53 \pm 0.76 \%$ and 8.40 $\pm 0.65 \%$ for each group, respectively. At week 52 , tofogliflozin produced in both groups significant reductions in body weight $(-1.52 \pm 0.207 \mathrm{~kg}$ and $-2.13 \pm 0.313 \mathrm{~kg}$, respectively) and $\mathrm{HbA}_{1 \mathrm{c}}$ levels $(-0.76 \pm 0.077 \%$ and $-0.73 \pm 0.102 \%$, respectively) [5].

Of great interest were also the results reported by Matsuba et al. [6]. The researchers documented that addition of tofogliflozin to DPP-4 treatment significantly improved carbohydrate metabolism 12 weeks after administration. More specifically, the researchers observed an increase in insulin sensitivity (as indicated by peripheral glucose uptake), with a mean change in the $M$ value of $0.90 \pm 1.28(p<0.05)$, along with a significant reduction in $\mathrm{HbA}_{1 c}$ by $1.05 \pm 0.47 \%$, in fasting plasma glucose by $2.17 \pm 1.70 \mathrm{mmol} / \mathrm{l}$ and in glycoalbumin by $4.21 \pm 2.07 \%$ ( $p<$ 0.001 for all parameters). The homeostasis model assessment of $\beta$-cell function index increased by $13.35 \pm 9.33(p<0.001)$. At the end of the study, the researchers also observed a decrease in body weight by

\author{
Corresponding author: \\ Dimitrios Ioannis Patoulias \\ Department of \\ Internal Medicine \\ General Hospital \\ 3 B M. Alexandrou St \\ 57010 Veria, Greece \\ Phone: +30 6946900777 \\ E-mail: dipatoulias@gmail. \\ com
}


$2.87 \pm 1.48 \mathrm{~kg}$ and in BMI by $1.11 \pm 0.57 \mathrm{~kg} / \mathrm{m}^{2}$, while they also reported a reduction of body fat mass by $1.33 \pm 0.99 \mathrm{~kg}$ and lean body mass by 1.54 $\pm 0.77 \mathrm{~kg}$ ( $p<0.001$ for all). Finally, the researchers observed a significant negative correlation between changes in the $M$ value and body fat mass $(r=-0.67, p=0.012)$, a finding highly indicative of the metabolic effects of tofogliflozin [6].

Significant were also the results reported by Tobe et al. in a similar study population. They observed that tofogliflozin significantly decreased $\mathrm{HbA}_{1 \mathrm{c}}$ levels, fasting plasma glucose levels and body weight, in all involved patients, who were divided into sub-groups based on their baseline insulin levels. However, they noted that the above improvements were greater in those patients who had higher baseline insulin levels. The latter group consisted of patients who were younger, had a shorter duration of diabetes, higher bodyweight and higher BMI at baseline, than the patients in the medium- and low-insulin groups [7].

Another recently published retrospective clinical study in 37 Japanese patients with type 2 diabetes mellitus, obese, with poor glycemic control (baseline BMI: $32.2 \pm 0.8 \mathrm{~kg} / \mathrm{m}^{2}$ and baseline $\mathrm{HbA}_{1 \mathrm{c}}$ levels: $8.3 \pm 0.2 \%$ ) and a mean duration of diabetes of $11.3 \pm 1.2$ years, proved that addition of tofogliflozin to the antidiabetic regimen of the above patients led to a significant decrease in both metabolic parameters. However, further analyses showed that tofogliflozin significantly reduced body fat mass, visceral fat area and soft lean mass, with a significant correlation between improvement in glycemic control and in measured body mass parameters, as indicated by univariate analyses. Thus, the authors reached the conclusion that tofogliflozin may be especially useful in a distinct phenotype of obese patients with short duration of diabetes [8].

A recent meta-analysis conducted by Cai et al. showed that body weight was significantly decreased in patients with type 2 diabetes who received SGLT-2 inhibitors at different doses, compared with placebo [9]. Treatment with tofogliflozin was associated with significant reduction in body weight $(-1.68,-2.15$ and $-2.35 \mathrm{~kg}$ for doses $10 \mathrm{mg} /$ day, $20 \mathrm{mg} /$ day and $40 \mathrm{mg} /$ day, respectively; $p<0.001)$. It is noteworthy that tofogliflozin studies included in the meta-analysis involved only the Asian population. Another significant finding was that, as shown in sub-group analysis, body weight reduction in patients with baseline $\mathrm{BMI}$ $<30 \mathrm{~kg} / \mathrm{m}^{2}$ was comparable with the corresponding reduction in patients with baseline $\mathrm{BMI} \geq 30$ $\mathrm{kg} / \mathrm{m}^{2}$. Despite the high heterogeneity of the results, it is clear that tofogliflozin induces a significant weight reduction, which makes this novel SGLT-2 inhibitor an attractive treatment option [9].
A closer look at the above findings leads to the conclusion that tofogliflozin seems very promising in Japanese patients with short duration of type 2 diabetes (as indicated by the correlation with baseline insulin levels in the study by Tobe et al.), inadequate glycemic control, and increased BMI levels at baseline, bordering on obesity. It seems that there is a distinct phenotype of patients who will benefit more from tofogliflozin therapy, an observation that needs to be verified in future trials involving patients of different races and maybe with lower baseline BMI levels.

\section{Conflict of interest}

The author declares no conflict of interest.

\section{References}

1. Zinman B, Wanner C, Lachin JM, et al.; EMPA-REG OUTCOME Investigators. Empagliflozin, cardiovascular outcomes, and mortality in type 2 diabetes. $\mathrm{N}$ Engl J Med 2015; 373: 2117-28.

2. Neal B, Perkovic V, Mahaffey KW, et al.; CANVAS Program Collaborative Group. Canagliflozin and cardiovascular and renal events in type 2 diabetes. $N$ Engl J Med 2017; 377: 644-57.

3. Hirose S, Nakajima S, Iwahashi Y, Seo A, Takahashi T, Tamori Y. Impact of the 8-week administration of tofogliflozin for glycemic control and body composition in Japanese patients with type 2 diabetes mellitus. Intern Med 2016; 55: 3239-45.

4. Suzuki K, Mitsuma Y, Sato T, Anraku T, Hatta M. Comparison of combined tofogliflozin and glargine, tofogliflozin added to insulin, and insulin dose-increase therapy in uncontrolled type 2 diabetes. J Clin Med Res 2016; 8: 805-14.

5. Terauchi Y, Tamura M, Senda M, Gunji R, Kaku K. Longterm safety and efficacy of tofogliflozin as add-on to insulin in patients with type 2 diabetes: results from a 52-week, multicentre, randomized, double-blind, open-label extension, phase 4 study in Japan (J-STEP/ INS). Diabetes Obes Metab 2018 Jan 5. doi: 10.1111/ dom.13213.

6. Matsuba R, Matsuba I, Shimokawa M, Nagai Y, Tanaka Y. Tofogliflozin decreases body fat mass and improves peripheral insulin resistance. Diabetes Obes Metab 2018 Jan 5. doi: 10.1111/dom.13211.

7. Tobe K, Suganami H, Kaku K. Sodium-glucose cotransporter 2 inhibitor, tofogliflozin, shows better improvements of blood glucose and insulin secretion in patients with high insulin levels at baseline. J Diabetes Investig 2017 Oct 15. doi: 10.1111/jdi.12761.

8. Kamei S, Iwamoto M, Kameyama M, et al. Effect of tofogliflozin on body composition and glycemic control in Japanese subjects with type 2 diabetes mellitus. J Diabetes Res 2018; 2018: 6470137.

9. Cai X, Yang W, Gao X, et al. The association between the dosage of SGLT2 inhibitor and weight reduction in type 2 diabetes patients: a meta-analysis. Obesity (Silver Spring) 2018; 26: 70-80. 